\title{
Endothelial VEGF Sculpts Cortical Cytoarchitecture
}

\author{
Suyan Li, ${ }^{1}$ Katharina Haigh, ${ }^{2,3,4}$ Jody J. Haigh, ${ }^{2,3,4 *}$ and Anju Vasudevan ${ }^{1 *}$ \\ ${ }^{1}$ Angiogenesis and Brain Development Laboratory, Division of Basic Neuroscience, McLean Hospital/Harvard Medical School, Belmont, Massachusetts \\ 02478, ${ }^{2}$ Vascular Cell Biology Unit, Department for Molecular Biomedical Research, VIB, B-9052 Ghent, Belgium, ${ }^{3}$ Department for Biomedical Molecular \\ Biology, Ghent University, B-9052 Ghent, Belgium, and ${ }^{4}$ Australian Centre for Blood Diseases, Monash University, Melbourne, VIC 3004, Australia
}

Current models of brain development support the view that VEGF, a signaling protein secreted by neuronal cells, regulates angiogenesis and neuronal development. Here we demonstrate an autonomous and pivotal role for endothelial cell-derived VEGF that has far-reaching consequences for mouse brain development. Selective deletion of Vegf from endothelial cells resulted in impaired angiogenesis and marked perturbation of cortical cytoarchitecture. Abnormal cell clusters or heterotopias were detected in the marginal zone, and disorganization of cortical cells induced several malformations, including aberrant cortical lamination. Critical events during brain development-neuronal proliferation, differentiation, and migration were significantly affected. In addition, axonal tracts in the telencephalon were severely defective in the absence of endothelial VEGF. The unique roles of endothelial VEGF cannot be compensated by neuronal VEGF and underscores the high functional significance of endothelial VEGF for cerebral cortex development and from disease perspectives.

\section{Introduction}

Vascular endothelial growth factor (VEGF or VEGF-A), a powerful secreted signaling protein, is indispensable for physiological angiogenesis (Carmeliet et al., 1996; Ferrara et al., 1996), vascular homeostasis (Lee et al., 2007), and pathological angiogenesis (Hicklin and Ellis, 2005). Current notions of brain development support the view that neuronal progenitors and/or neurons are the principal source of VEGF (Ogunshola et al., 2000) that act by binding to VEGF receptors expressed on vascular endothelial cells (Mustonen and Alitalo, 1995) to stimulate angiogenesis. High VEGF expression by neuronal progenitors in the embryonic ventricular zone (VZ) induces angiogenesis by paracrine mechanisms (Breier et al., 1995; Ogunshola et al., 2002). Consequently, conditional deletion of Vegf from neuronal progenitors resulted in impaired angiogenesis and severe disruption of neuronal development at early embryonic stages (Haigh et al., 2003; Raab et al., 2004). Although VEGF expression in endothelial cells has been reported during brain development (Virgintino et al., 2003) and plays an important role in adult endothelial homeostasis (Lee et al., 2007), its role during embryonic brain development remains unknown. Our studies have challenged notions of cerebral vascularization, which imply that blood vessels sprout passively to meet metabolic demands of growing neuronal populations

\footnotetext{
Received April 1, 2013; revised July 18, 2013; accepted Aug. 9, 2013.

Author contributions: J.J.H. and A.V. designed research; S.L. and K.H. performed research; J.J.H. and A.V. contributed unpublished reagents/analytic tools; S.L., K.H., J.J.H., and A.V. analyzed data; J.J.H. and A.V. wrote the paper.

This work was supported by a National Alliance for Research on Schizophrenia and Depression Young Investigator Award and National Institutes of Health Grants R21NS064386 and R01NS073635 to A.V.

The authors declare no competing financial interests.

*J.J.H. and A.V. contributed equally to this work.

Correspondence should be addressed to Dr. Anju Vasudevan, Angiogenesis and Brain Development Laboratory,

Division of Basic Neuroscience, McLean Hospital/Harvard Medical School, 115 Mill Street, Belmont, MA 02478.

E-mail: avasudevan@mclean.harvard.edu.

DOI:10.1523/JNEUROSCI.1368-13.2013

Copyright $\odot 2013$ the authors $\quad 0270-6474 / 13 / 3314809-07 \$ 15.00 / 0$
}

and offered new perspectives on intrinsic regulation of angiogenesis in the embryonic telencephalon (Vasudevan et al., 2008). Periventricular vessels are distinct from pial vessels based on anatomical location, growth patterns, and gene expression profiles (Vasudevan et al., 2008; Won et al., 2013). In addition, the periventricular vascular network provides support and critical guidance cues to instruct neuronal migration in the developing telencephalon (Won et al., 2013). Because the propagation of the periventricular angiogenesis gradient corresponds to the neurogenetic gradient with respect to direction (Vasudevan et al., 2008) and the gradient of VEGF expression mirrors the neurogenetic gradient (Virgintino et al., 2003), we selectively deleted Vegf from endothelial cells to investigate the functional significance of endothelial cell-derived VEGF in the embryonic telencephalon. Our results unveil a unique role for endothelial VEGF in regulating cortical histogenesis and highlight the complexity of neurovascular cross talk and interactions in time and space that is of great significance for neuronal development and disease.

\section{Materials and Methods}

Animals. To selectively delete Vegfin endothelial cells, Tie2-cre transgenic mice (males) were crossed to Vegf floxed (Vegf flfl $)$ mice (females) to generate Tie2-cre; Vegf $f^{f l+}$ mice (males). These were further crossed with $V e g f^{f l f l}$ mice (females) to obtain the Vegf conditional knock-out, hereafter referred to as $V e g f^{\Delta T i e 2-C r e}$ mice. The Tie2-cre transgene is known for uniform expression of the cre-recombinase in endothelial cells during embryogenesis (Schlaeger et al., 1997; Koni et al., 2001). All animal procedures were approved by the animal care and use committee of Ghent University.

Bromodeoxyuridine (BrdU) labeling. A single BrdU injection $(50 \mu \mathrm{g} / \mathrm{g})$ was administered to pregnant dams carrying E13 or E17 mice. Embryonic brains were removed after $2 \mathrm{~h}$, immersed in zinc fixative (BD Biosciences PharMingen) for $24 \mathrm{~h}$, and processed for paraffin wax histology. BrdU immunohistochemistry was performed on coronal, $20 \mu \mathrm{m} \mathrm{sec-}$ tions. Double labeling was performed with a mouse monoclonal anti- 

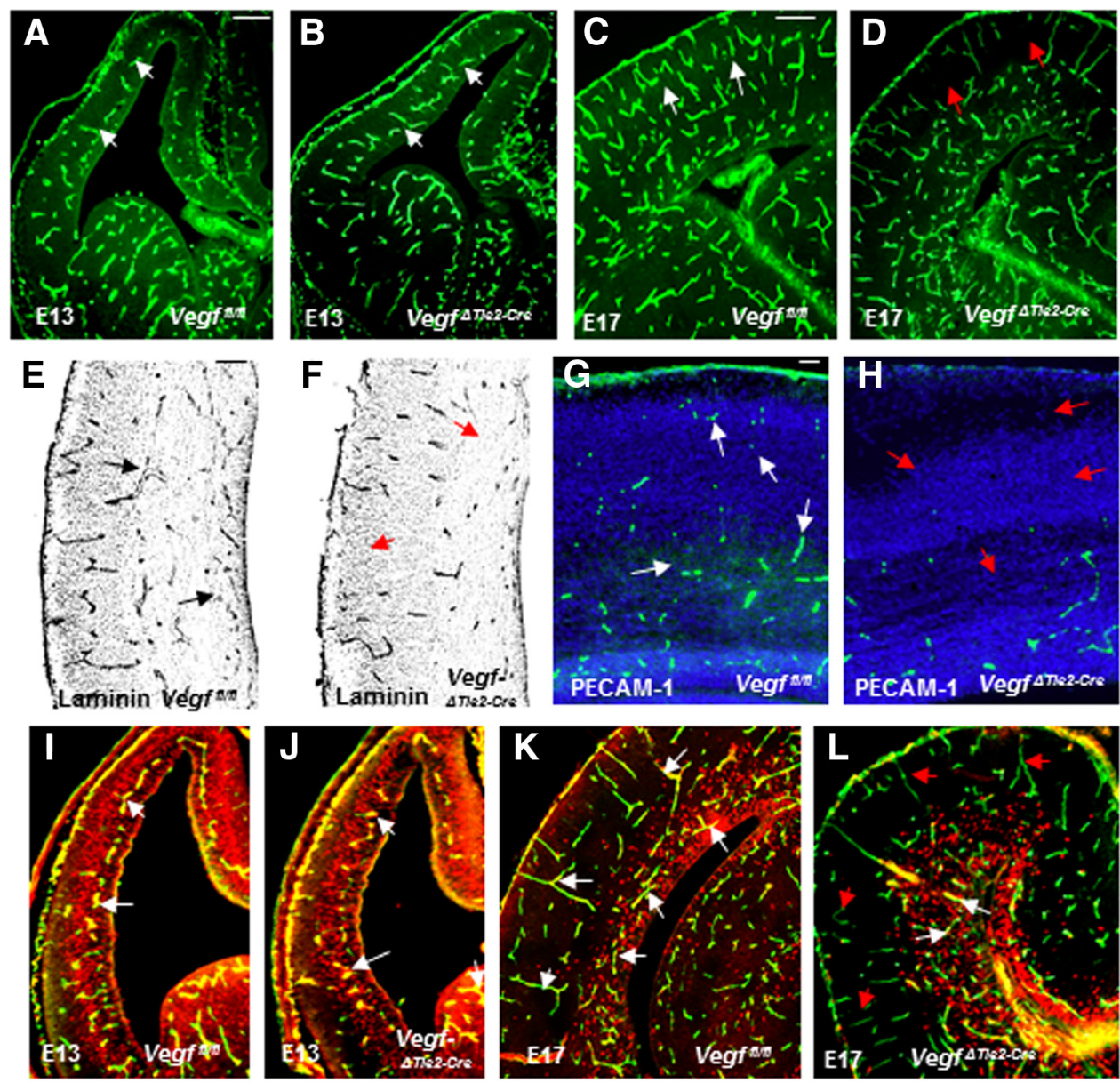

M

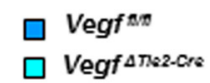

$\mathbf{N}$
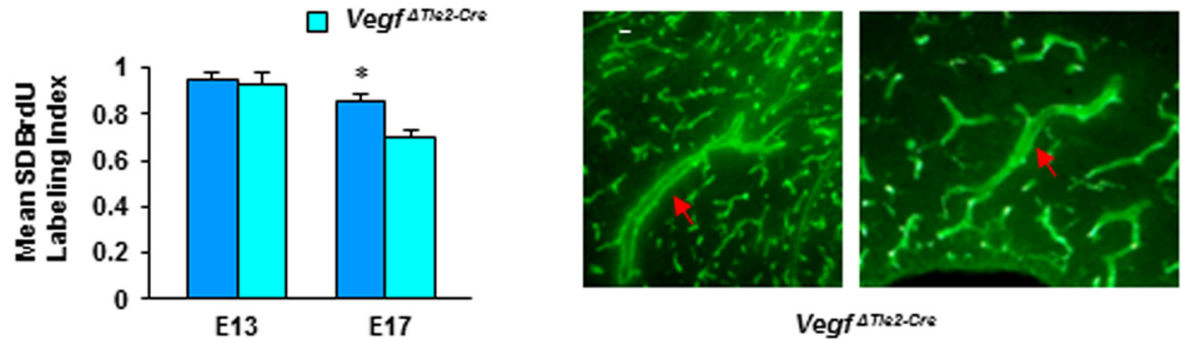

Figure 1. Impaired angiogenesis in Vegf ${ }^{\Delta T i e 2-C r e}$ embryos. $\boldsymbol{A}, \boldsymbol{B}$, Isolectin $B 4^{+}$periventricular vessel gradient (white arrows) in E13 Vegf ${ }^{f / f l}(\boldsymbol{A})$ and Vegf ${ }^{\Delta \text { Tiez-Cre }}(\boldsymbol{B})$ telencephalon. $\boldsymbol{C}, \boldsymbol{D}$, Fewer isolectin $B 4^{+}$vessels in E17 Vegf ${ }^{\Delta \text { Tiez-Cre }}$ pallium $\left(\boldsymbol{D}\right.$, red arrows) compared with Vegf ${ }^{f / f t}$ pallium ( $\boldsymbol{C}$, white arrows). $\boldsymbol{E}, \boldsymbol{F}$, Fewer laminin ${ }^{+}$vessels in E17 Vegf ${ }^{\Delta \text { tiez-Cre }}$ pallium $(\boldsymbol{F}$, red arrows) compared with Vegf $f^{f / f f}$ pallium (E, black arrows). $\mathbf{G}, \boldsymbol{H}$, Fewer PECAM-1 ${ }^{+}$endothelial cells in E17 Vegf ${ }^{\Delta T i e z-\text { cre }}$ pallium ( $\boldsymbol{H}$, red arrows) compared with Vegf $f^{f / f l}$ pallium $(\boldsymbol{G}$, white arrows). $\boldsymbol{I}, \boldsymbol{J}$, Double labeling of endothelial cells with isolectin B4 and BrdU (white arrows point to colabel in yellow) shows no differences in endothelial cell proliferation in E13 Vegf ${ }^{f / f f}$ and Vegf ${ }^{\Delta T i e z-C r e}$ telencephalon. $\boldsymbol{K}$, Endothelial cells proliferate in neurogenic and postmitotic regions of E17 Vegf flffl telencephalon (white arrows point to coexpressing cell nuclei that appear in yellow). $\boldsymbol{L}$, Endothelial cells proliferate only in neurogenic regions (white arrows), and there is lack of BrdU-isolectin B4 colabel in postmitotic regions (red arrows) of Vegf ${ }^{\Delta T \text { Tiez-Cre }}$ telencephalon. $M$, Endothelial cell BrdU labeling index was comparable in E13 Vegf $f^{f / f l}$ and Vegf ${ }^{\Delta \text { Tiez-Cre }}$ telencephalon $(n=8$, mean $\pm S D, p>0.05)$ and significantly decreased in E17 Vegf ${ }^{\Delta \text { Tiez-Cre }}$ telencephalon compared with Vegf ${ }^{f / f l}$

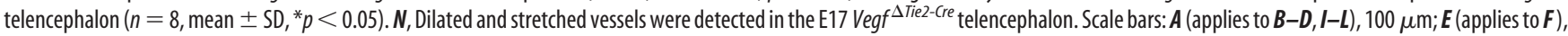
$75 \mu \mathrm{m} ; \boldsymbol{G}$ (applies to $\boldsymbol{H}$ ), $50 \mu \mathrm{m} ; \boldsymbol{N}, 25 \mu \mathrm{m} . n=8$.

body to $\operatorname{BrdU}(1: 75,347580$, BD Biosciences PharMingen) and biotinylated isolectin B4 (1:40, L2140, Sigma).

Histology and Immunohistochemistry. E13, E15, and E17 brains were fixed in zinc fixative (BD Biosciences PharMingen) for $24 \mathrm{~h}$ and processed for paraffin histology. Histological stainings with hematoxylin (Vector Laboratories) and eosin (Sigma) were performed on $8 \mu \mathrm{m}$ coronal sections, and lectin histochemistry (with biotinylated isolectin B4, 1:40, Sigma) as well as immunohistochemistry were performed on $20 \mu \mathrm{m}$ sections. Primary antibodies used for immunohistochemistry were as follows: mouse monoclonal antibody to RC2 (1:5, MAB5740, Millipore), goat polyclonal antibody to CHL1 (1:50, AF2147, R\&D Systems), rat monoclonal antibody to CD31/PECAM-1 (1:50, 550274, BD Biosciences PharMingen), and rabbit polyclonal antibodies to laminin (1:10, L9393, Sigma), Ki67 (1:30, HPA000451, Sigma), microtubule associated protein 2 (MAP2; 1:50, M3696, Sigma), Tbr1 (1:25, ab31940, Abcam), GAD65/67 (1:50, AB1511, Millipore), calbindin (1:30, AB1778, Millipore), calretinin $(1: 30,7699 / 3 \mathrm{H}$, Swant), followed by secondary detection with AlexaFluor conjugates (Invitrogen). DAPI (Vector Laboratories) was used to label nuclei. High-magnification images were obtained from an FSX100 microscope (Olympus).

Morphometry. A stereological point grid was superimposed on digital images of biotinylated isolectin-B $4^{+}$vessels. The ratio between points 

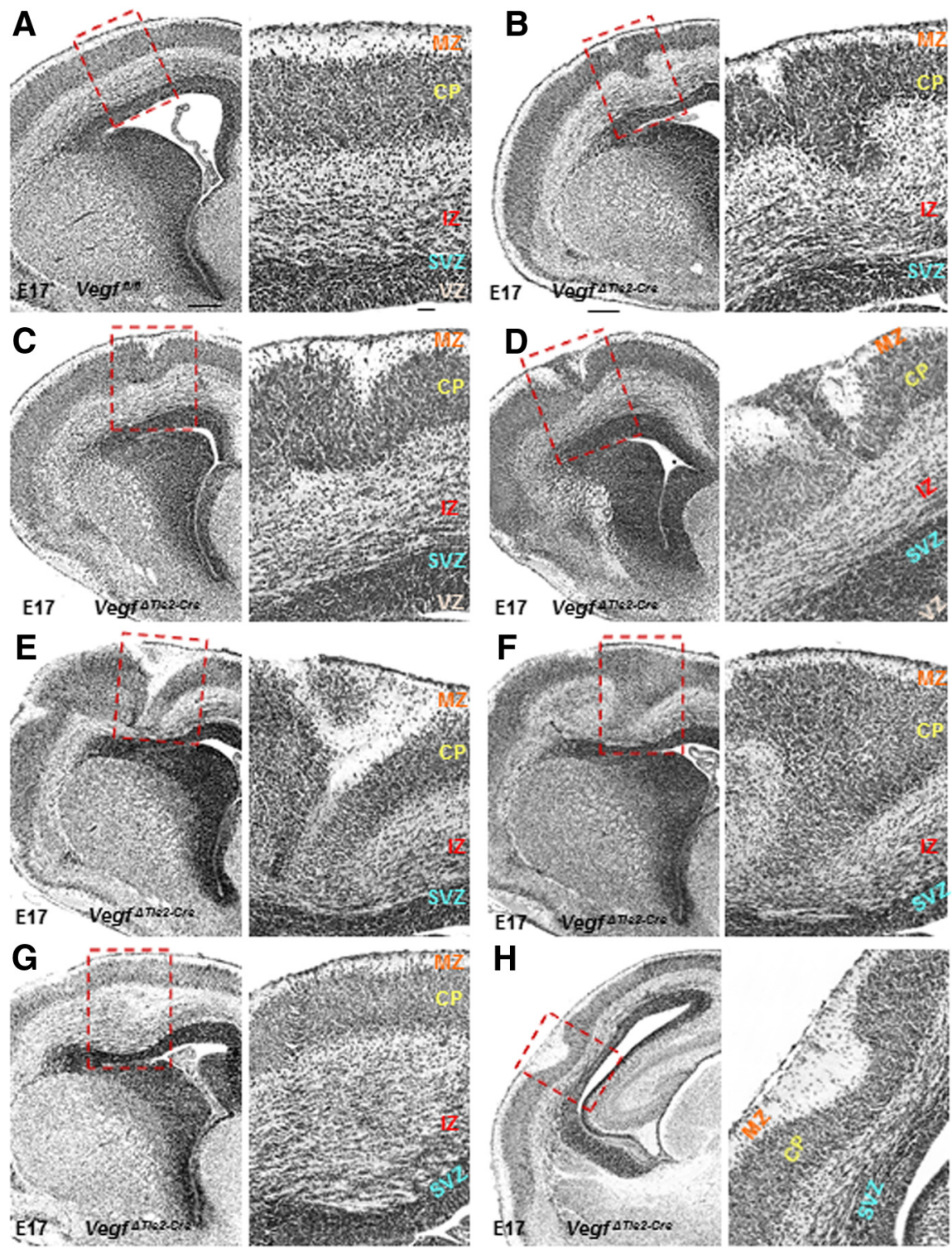

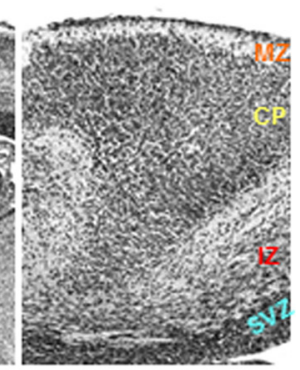

$\mathbf{H}$
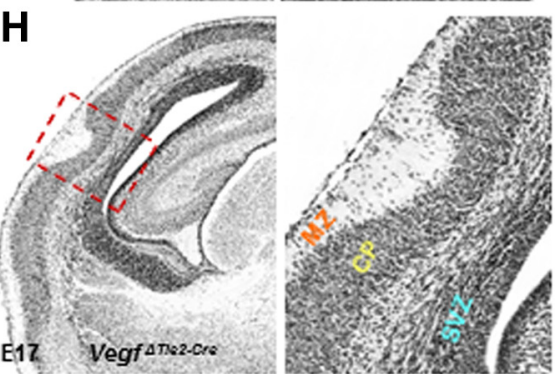

Figure 2. Cortical malformations in E17 Vegf ${ }^{\Delta T i e 2-C r e}$ embryos. $\boldsymbol{A}$, Hematoxylin and eosin staining showed ordered cortical laminar organization in Vegf ${ }^{f / f l}$ embryos. $\boldsymbol{B}-\boldsymbol{H}$, Hematoxylin and eosin staining revealed marked perturbation of cortical cytoarchitecture in Vegf ${ }^{\Delta T i e 2-C r e}$ embryos. $\boldsymbol{A}-\boldsymbol{H}$, Boxed areas have been magnified $(60 \times)$ and cortical layers labeled. Scale bars: $\boldsymbol{A}$ (applies to $\boldsymbol{B}-\boldsymbol{H}$ ), $100 \mu \mathrm{m}$; insets $(\boldsymbol{A}-\boldsymbol{H}), 50 \mu \mathrm{m} . n=8$.

falling on blood vessels and on brain tissue was calculated for each section, and average values were obtained for a given brain.

Statistics. Statistical significance of differences between groups was analyzed by two-tailed Student's $t$ test (Prism; GraphPad software). Results were expressed as mean $\pm \mathrm{SD}$, and statistical significance was reported at $p<0.05$.

\section{Results}

Impaired angiogenesis in Vegf ${ }^{\Delta T i e z-C r e}$ embryos

To test whether deletion of Vegf from endothelial cells affected telencephalic angiogenesis, we first investigated vascular profiles in Vegf flfl (wild-type littermates) and Vegf ${ }^{\Delta \text { Tiez-Cre }}$ embryos at early (E13) and late (E17) embryonic stages. By E13, the periventricular vessel gradient from ventral to dorsal telencephalon was clearly visible in Vegf ${ }^{\Delta \text { Tie2-Cre }}$ embryos (Fig. $1 B$ ). Periventricular vessel numerical density (expressed as percentage) showed no significant differences in E13 Vegf flfl $(15.54)$ and Vegf $f^{\Delta \text { Tiez-Cre }}$ (14.79) telencephalon (Fig. $1 A, B, n=8$, mean $\pm \mathrm{SD}, p>0.05$ ).
However, by E17, vessel density was significantly reduced in $V e g f^{\Delta T i e 2-C r e}$ telencephalon (21.65) compared with Vegf flfl telencephalon (26.75) (Fig. $1 C, D, n=8$, mean $\pm \mathrm{SD}, p<0.05)$. Reduction in vessel densities was more prominent in the dorsal telencephalon (pallium) of $\mathrm{Veg} f^{\Delta \mathrm{Tie} 2-\mathrm{Cre}}$ embryos (Fig. 1D). Labeling with multiple markers of vessel components, laminin (Fig. $1 E, F$ ) and CD31/PECAM-1 (Fig. 1G,H) confirmed the reduction in E17 Vegf ${ }^{\Delta \text { Tie2-Cre }}$ pallium. Similar to vessel densities, endothelial cell proliferation was comparable in E13 Vegf flffl and Vegf $f^{\Delta T i e 2-C r e}$ telencephalon (Fig. 1I,J,M). Endothelial cell proliferation continued independent of neuroepithelial cell proliferation in both neurogenic and postmitotic regions of Vegf ${ }^{f l f l}$ telencephalon at E17 (Fig. $\left.1 K\right)$. Endothelial cell proliferation in postmitotic regions was affected such that the $2.0 \mathrm{~h}$ BrdU labeling index was significantly reduced in Vegf $f^{\Delta \text { Tiez-Cre }}$ telencephalon at E17 (Fig. $1 L, M)$. Dilated and abnormally stretched vessels were also observed in the Vegf ${ }^{\Delta T i e 2-C r e}$ telencephalon (Fig. 1N).

\section{Cortical malformations in} Vegf $f^{\Delta \text { Tie2-Cre }}$ embryos

The morphology of Vegf ${ }^{\Delta \text { Tie2-Cre }}$ telencephalon at E13 appeared normal in histological stainings and comparable to Vegf $f^{f / f l}$ telencephalon (data not shown). But, by E17, while ordered cortical laminar organization was observed in $V e g f^{f l / f l}$ embryos (Fig. 2A), deletion of endothelial VEGF had far-reaching effects with marked perturbation of cortical cytoarchitecture in $V e g f^{\Delta \text { Tiez-Cre }}$ embryos (Fig. $2 B-H$ ). The malformations included the following: polymicrogyria overlying heterotopic cortical plate (CP) (Fig. 2B,C), marginal zone (MZ) heterotopias (Fig. 2D), cortical dysplasia, including split CP mislocated to intermediate zone (IZ) combined with MZ heterotopia and reduced subventricular zone (SVZ) (Fig. 2E), dispersion of CP cells into deeper layers of IZ (Fig. 2F), and abnormally enlarged IZ (Fig. 2G), all of which caused severe disruption of cortical lamination. These defects were present both at rostral (Fig. $2 B-G$ ) and caudal (Fig. $2 H$ ) levels of $V e g f^{\Delta T i e 2-C r e}$ telencephalon. Our data offer novel evidence to the important role of endothelial cell-derived VEGF in periventricular angiogenesis with significant consequences for cortical development.

\section{Perturbed neuronal proliferation, migration, and lamination} in Vegf $f^{\Delta \text { Tie2-Cre }}$ telencephalon

Because E15 is an age midway through mouse cortical neurogenesis and migration, we investigated whether vascular and neuronal-specific defects could be observed by this stage. Endothelial cells/vessels in the $V e g f^{\Delta \text { Tie2-Cre }}$ pallium were reduced as observed by labeling with PECAM-1 (Fig. $3 A, B$ ), laminin (Fig. $3 C, D$ ), and isolectin B4 (Fig. $3 E, F$ ) markers. To define the iden- 

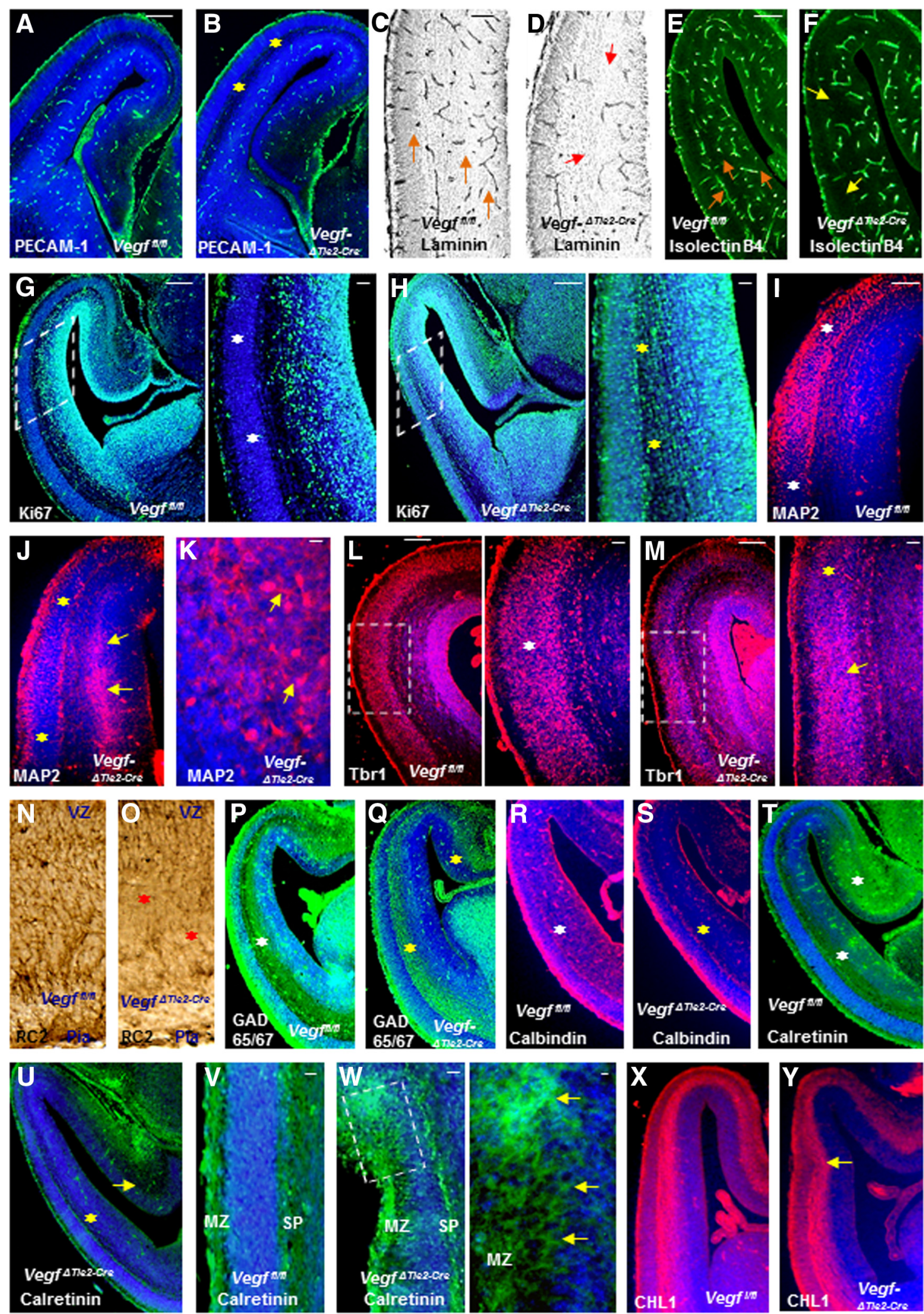

Figure 3. Perturbation of vascular and neuronal development in E15 Vegf ${ }^{\Delta \text { Tiez-Cre }}$ telencephalon. $\boldsymbol{A}-\boldsymbol{F}$, Reduced PECAM- $1^{+}$endothelial cells $\left(\boldsymbol{B}\right.$, yellow asterisk), laminin ${ }^{+}$vessels $(\boldsymbol{D}$, red arrows), and isolectin $B 4^{+}$vessels ( $\boldsymbol{F}$, yellow arrows) in $\mathrm{E} 15$ Vegf $^{\Delta \text { tiez-cre }}$ pallium $(\boldsymbol{B}, \boldsymbol{D}, \boldsymbol{F})$ compared with Vegf $f^{f / f f}$ pallium $(\boldsymbol{A}, \boldsymbol{C}, \boldsymbol{E})$. $\boldsymbol{C}, \boldsymbol{E}$, Orange arrows indicate well-formed vascular network in Vegf $f^{f / f f}$ pallium. $\mathbf{G}, \boldsymbol{H}$, Ki67 labeling marks the proliferating progenitor pool in Vegf $f^{f / f}$ telencephalon $(\boldsymbol{G})$, whereas Ki67-positive progenitors spanned the entire pallium of Vegf ${ }^{\Delta T i e z-(r e}$ embryos $(\boldsymbol{H})$. Boxed regions were magnified; white asterisks indicate Ki67-negative $(\mathbf{P}(\boldsymbol{G})$, and yellow asterisks indicate abnormal proliferation $(\boldsymbol{H}) . \boldsymbol{I}, \boldsymbol{J}$, MAP2 immunoreactivity reveals normal neuronal differentiation zone of E15 Vegf ${ }^{f / f l}$ pallium (I, white asterisks), which is reduced in Vegf ${ }^{\Delta \text { Tiez-cre }}$ pallium (J, yellow asterisks). A band of MAP2 ${ }^{+}$cells were abnormally positioned in the Vegf ${ }^{\Delta T i e z-\text {-re }}$ IZ-SVZ (J, yellow arrows). $\boldsymbol{K}$, High-magnification image of MAP2 ${ }^{+}$neurons in the Vegf ${ }^{\Delta T i e z-c r e}$ SVZ. $L, \boldsymbol{M}$, Tbr1 immunoreactivity in E15 Vegf ${ }^{\text {fl/ff }}(\boldsymbol{L})$ and Vegf ${ }^{\Delta \text { Tiez-Cre }}(\boldsymbol{M})$ pallium. Boxed regions are magnified. The uniform arrangement of $\mathrm{CP}$ cells in Vegf ${ }^{f / f f}$ pallium ( $\boldsymbol{L}$, white asterisk) was altered in Vegf ${ }^{\Delta \text { Tiez-Cre }}$ pallium with abnormally thicker $(\boldsymbol{M}$, yellow arrow) and thinner $(\boldsymbol{M}$, yellow asterisk) cell arrangement in the CP. N, O, RC2 immunostaining reveals continuous radial glial fibers extending from VZ to pial surface in Vegf ${ }^{\mathrm{f} / f / f}$ pallium $(\boldsymbol{N})$ and shortened radial glial (Figure legend continues.) 
tity of the cells that accounts for the defective cortical cytoarchitecture, we examined the expression of markers specific for neuroepithelial precursors and differentiating neurons. Progenitor cells in neurogenic regions around the lateral ventricles were identified in Vegf $f^{f l f l}$ telencephalon by Ki67 immunoreactivity (Fig. 3G). In contrast, abnormally proliferating progenitors were observed outside the neurogenic zone along the full length of the $V e g f^{\Delta T i e 2-C r e}$ pallium (Fig. $3 H$ ). Spatial patterns of neuronal differentiation were examined by expression of MAP2 and cortical neuron population marker Tbr1, which marks CP, subplate (SP), and layer VI in wild-type embryos (Fig. 3I-M). MAP2immunoreactive postmitotic neurons were detected within the SVZ in Vegf ${ }^{\Delta T i e z-C r e}$ embryos, revealing evidence of perturbed neuronal migration (Fig. $3 \mathrm{~J}, \mathrm{~K}$ ). Tbr1 immunoreactivity revealed a continuous uniform band of Tbr1-positive cells in the CP of E15 Vegf flff embryos (Fig. $3 L$ ). On the other hand, abnormally thicker and thinner bands of Tbr1-positive cells were detected in the CP of Vegf ${ }^{\Delta \text { Tiez-Cre }}$ embryos (Fig. $3 M$ ). The radial glial scaffold that extends fibers from ventricular to pial surface in Vegf $f^{f l f l}$ embryos (Fig. $3 N$ ) and is essential for guiding migrating cortical neurons was shortened and disrupted in Vegf ${ }^{\Delta T i e 2-C r e}$ embryos (Fig. 3O). We also examined the tangential stream of GABA neurons that migrate from basal to dorsal telencephalon in $V e g f^{f l f l}$

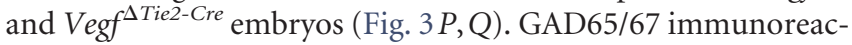
tivity depicted a reduced GABA neuron stream in the dorsal telencephalon of Vegf ${ }^{\Delta T i e 2-C r e}$ embryos (Fig. 3Q). To identify whether specific subsets of GABA neurons were affected, we used calbindin and calretinin immunohistochemistry (Fig. 3R-U). Calbindin-positive and calretinin-positive neurons were significantly reduced in $V e g f^{\Delta T i e 2-C r e}$ pallium (Fig. $3 S, U$ ). Several layers of the dorsomedial telencephalon labeled heavily for calretinin in $V e f^{f l / f l}$ embryos (Fig. 3T); in contrast, calretinin-positive cells were significantly reduced dorsomedially in Vegf ${ }^{\Delta T i e 2-C r e}$ embryos (Fig. 3U). Calretinin-positive cells formed a single continuous row and labeled Cajal-Retzius cells of the MZ and SP in Vegf $f^{f l / f l}$ embryos (Fig. $3 V$ ). The uniform layer of the MZ in Vegf $f^{\Delta \text { Tiez-Cre }}$ telencephalon was disturbed at places, calretinin-positive cells were seen outside the MZ, and calretinin-labeled fibers in the SP were thinner (Fig. $3 W)$. Axonal tracts labeled by neural cell adhesion molecule, CHL1 (Fig. $3 X, Y$ ) occasionally narrowed and appeared displaced in the $V_{e g} f^{\Delta T i e 2-C r e}$ dorsal telencephalon at this stage (Fig. $3 Y$ ).

These defects in neuronal proliferation, differentiation, and lamination observed in E15 dorsal telencephalon of Vegf $f^{\Delta \text { Tiez-Cre }}$ embryos became marked by E17 and reflected diversely in a wide range of severe cortical malformations (Fig. 4A-F). Ki67-positive proliferating progenitors were observed infiltrating the CP ectopias in E17 Vegf ${ }^{\Delta T i e 2-C r e}$ embryos (Fig. 4B). Breakdown of pial

\footnotetext{
(Figure legend continued.) processes in Vegf ${ }^{\Delta \text { tiez-Cre }}$ pallium $(\mathbf{0}$, red asterisks). $\boldsymbol{P}, \mathbf{Q}$, GAD65/67 immunoreactivity shows decreased stream of GABA neurons in Vegf ${ }^{\Delta T i e 2-C r e}$ pallium extending to the medial edge ( $\boldsymbol{Q}$, yellow asterisks) compared with Vegf ${ }^{f / f l}$ pallium $(\boldsymbol{P}$, white asterisks). $\boldsymbol{R}-\boldsymbol{U}$, Calbindin ${ }^{+}$and calretinin ${ }^{+}$neurons were decreased ( $\boldsymbol{S}, \boldsymbol{U}$, yellow asterisks) in E15 Vegf ${ }^{\Delta \text { Tiez-Cre }}$ pallium compared with Vegf ${ }^{f / f f}$ pallium $(\boldsymbol{R}, \boldsymbol{I}$, white asterisks). Calretinin immunoreactivity was decreased in the hippocampal primodium of Vegf ${ }^{\Delta \text { tiez-Cre }}$ embryos $(\boldsymbol{U}$, yellow arrow). $\boldsymbol{V}, \boldsymbol{W}$, Uniform calretinin labeling in $\mathrm{MZ}$ and SP of Vegf ${ }^{\text {ff/fl }}$ embryos $(\boldsymbol{V})$ and irregular calretinin labeling outside MZ in Vegf ${ }^{\Delta \text { Tiez-cre }}$ telencephalon $(\boldsymbol{W})$. $\boldsymbol{W}$, The boxed region was magnified, and yellow arrows point to calretinin ${ }^{+}$cells outside MZ. X, Y, CHL1 immunostaining reveals axonal tracts in Vegf $f^{f / f f}(\boldsymbol{X})$ and Vegf ${ }^{\Delta \text { Tiez-Cre }}(\boldsymbol{\eta})$ pallium. $\boldsymbol{Y}$, Yellow arrow points to displaced axonal tract. DAPI (blue) was used to label nuclei $(\boldsymbol{A}, \boldsymbol{B}, \mathbf{G}-\boldsymbol{M}, \boldsymbol{P}-\boldsymbol{\eta})$. Scale bars: $\boldsymbol{A}$ (applies also to $\boldsymbol{B}, \boldsymbol{E}-\boldsymbol{J}, \boldsymbol{L}, \boldsymbol{M}, \boldsymbol{P}-\boldsymbol{U}, \boldsymbol{X}, \boldsymbol{\eta}$ ), $100 \mu \mathrm{m} ; \boldsymbol{C}$ (applies also to $\boldsymbol{D}$ ), $75 \mu \mathrm{m} ; \boldsymbol{N}$ (applies to $\mathbf{O}$, $\boldsymbol{V}, \boldsymbol{W}$ ), $50 \mu \mathrm{m}$; magnified inset in $\boldsymbol{G}$ (applies to magnified insets in $\boldsymbol{H}, \boldsymbol{L}, \boldsymbol{M}$ ), $50 \mu \mathrm{m} ; \boldsymbol{W}$ (magnified inset), $25 \mu \mathrm{m} . n=8$.
}

membrane with concurrent neuronal overmigration as well as disruption of the VZ continuity was particularly obvious (Fig. $4 B$ ). Abnormal crests and grooves of MAP2-positive cells revealed intense polymicrogyria in E17 Vegf $f^{\Delta T i e 2-C r e}$ telencephalon (Fig. 4D). Tbr1 immunostaining showed split CP with the two edges of the overextended CP misplaced into SVZ-IZ regions (Fig. $4 F$ ). These results reveal the crucial role of endothelial VEGF that impacts neuronal progenitors, neurons, and radial glia in the developing telencephalon.

\section{Disturbed axonal tracts in Vegf $f^{\Delta T i e 2-C r e}$ embryos}

Axonal fiber tracts containing cortical efferent and afferent fibers innervated the internal capsule in E17 Vegf $f^{\Delta \text { Tie2-Cre }}$ embryos, and a loss of trajectory of axon tracts was not observed. However, axon tracts labeled with CHL1 were severely defective at all rostrocaudal levels in the E17 Vegf $f^{\Delta T i e 2-C r e}$ telencephalon (Fig. 4G$Z$ ). In contrast to the normal location of subcortical axonal fiber tracts just beneath the CP in Vegf fl/fl embryos, in Vegf $f^{\Delta T i e 2-C r e}$ embryos, the axon tracts thinned severely at places (Fig. $4 H, N, T, V$ ) or abnormally thickened and extended into CP (Fig. $4 N, T, V, X, Z)$. Fiber tracts that decussate at the midline, most notably the corpus callosum and anterior commissure, were affected in Vegf ${ }^{\Delta \text { Tie2-Cre }}$ embryos (Fig. $3 L, P$ ). Although corpus callosum fibers crossed the midline in Vegf fl/fl embryos (Fig. $3 K$ ), the callosal projections failed to show decussation in $V e g f^{\Delta T i e 2-C r e}$ embryos and stopped abruptly at the midline (Fig. $3 L$ ). Although the anterior commissure crossed at two levels along the rostrocaudal axis in $V e g f^{f l f l}$ embryos, the anterior commissure crossed at the midline only at the more caudal level, indicative of a thinner commissural tract in Vegf ${ }^{\Delta T i e 2-C r e}$ embryos (Fig. 3O-R). Thus, development of the major axon systems of the telencepha-

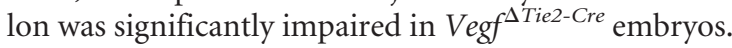

\section{Discussion}

Our results uncover endothelial VEGF's novel and critical role in molding the development of periventricular vessels, neuroepithelial cells, neurons, and eventually the telencephalic architecture. At early embryonic stages (E9-E13), neuronal VEGF signaling is the primary player regulating telencephalic development. By E15, when vessel density increases along with concurrent expansion of the cortex, endothelial VEGF becomes critical for ongoing developmental processes. Telencephalic angiogenesis was affected along with significant consequences for neuronal development. These results lay the basis for future studies investigating detailed mechanisms mediated by endothelial VEGF. It would also be interesting to investigate what different isoforms of endothelial VEGF are secreted and to what degree these isoforms specifically affect vascular and/or neuronal development.

The Tie2-Cre line used in this analysis has also been shown to be active in the hematopoietic system (Goossens et al., 2011), and previous reports have demonstrated that macrophage sources of VEGF as well as other angiogenic factors play important roles in vessel anastomosis during early vessel patterning in the developing CNS (Fantin et al., 2013). However, up until E13, we could not detect any changes in the vasculature associated with endothelial loss of VEGF and only by E15 were changes in vessel patterning observed. Microglia (the macrophage population of the CNS) increases significantly in number after birth concurrent with myelination events during postnatal brain development (Erblich et al., 2011). Despite loss of microglia, mouse embryonic forebrain development has been reported to be normal (Erblich et al., 2011). In addition, in the embryonic telencephalon, up until E17, microglia have been reported to be either absent from 

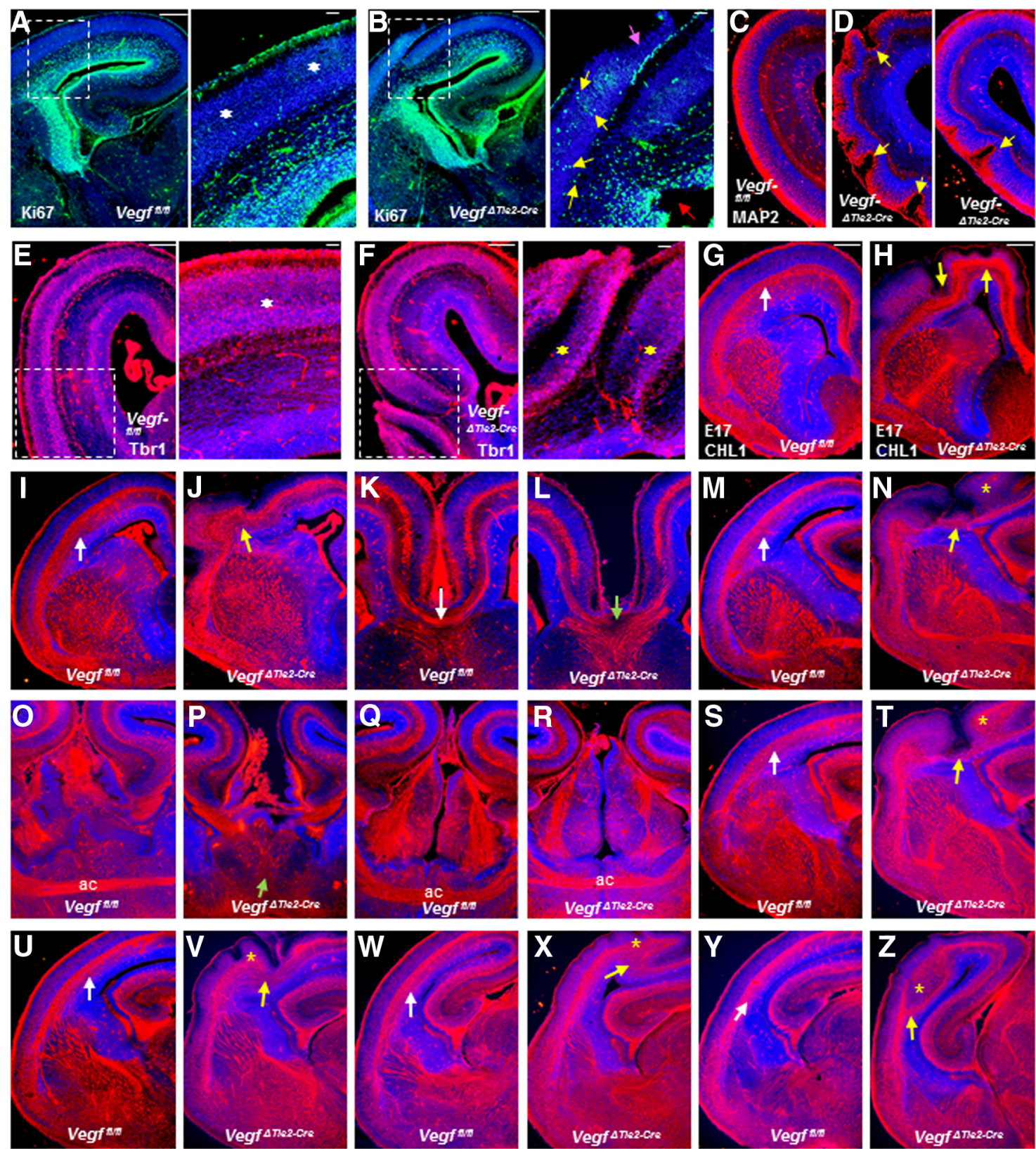

Figure 4. Marked disturbance of neuronal development and axonal tracts in E17 Vegf ${ }^{\Delta T i e 2-C r e}$ embryos. $\boldsymbol{A}, \boldsymbol{B}$, Ki67 labeling of E17 Vegf ${ }^{f l / f l}(\boldsymbol{A})$ and Vegf ${ }^{\Delta \text { Tiez-Cre }}(\boldsymbol{B})$ telencephalon. Boxed regions were magnified. $A$, Inset, White asterisks indicate Ki67-negative CP. $B$, Inset, Yellow arrows indicate abnormally proliferating Ki67-positive cells in a cortical ectopia. Red arrow points to a disrupted site along the ventricular surface. Pink arrow points to DAPI-labeled ectopia breaching pial surface. $\boldsymbol{C}, \boldsymbol{D}$, MAP2 labeling revealing normal differentiation zone in Vegf ${ }^{f / / f l}$ pallium $(\boldsymbol{C})$ and abnormal differentiation in Vegf ${ }^{\Delta \text { tiez-Cre }}$ pallium (D, yellow arrows). $\boldsymbol{E}, \boldsymbol{F}$, Tbr1 immunoreactivity in Vegf ${ }^{f l / f l}(\boldsymbol{E})$ and Vegf ${ }^{\Delta \text { tiez-Cre }}(\boldsymbol{F})$ embryos. Boxed regions were magnified, revealing well-organized $C P$ in Veg $f^{f / f t}$ embryos ( $\boldsymbol{E}$, white asterisk) and disrupted (P arrangement in Vegf ${ }^{\Delta T i e z-C r e}$ embryo ( $\boldsymbol{F}$, yellow asterisks). $\mathbf{G}-\boldsymbol{Z}$, CHL1 labeling of axonal tracts in Vegf ${ }^{f / f t}$ and Vegf ${ }^{\Delta / i e 2-C r e}$ embryos along the rostrocaudal axis. White arrows indicate well-formed axon tracts in sections from Vegf $f^{f / f l}$ embryos $(\boldsymbol{G}, \boldsymbol{I}, \boldsymbol{M}, \boldsymbol{S}, \boldsymbol{U}, \boldsymbol{W}, \boldsymbol{\eta}$. Axon tracts were severely disturbed in corresponding sections from

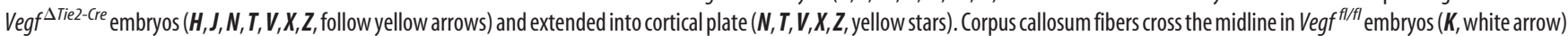
but stop abruptly at the midline in Vegf ${ }^{\Delta T i e z-C r e}$ embryos ( $L$, green arrow). The two limbs of the anterior commissure (ac) cross at the midline in Vegf ${ }^{f / f l}$ embryos at two levels along the rostrocaudal axis $(\boldsymbol{O}, \mathbf{Q})$. Crossing of ac at the midline does not happen at the rostral level in Vegf ${ }^{\Delta T i e 2-C r e}$ embryos $(\boldsymbol{P}$, green arrow), but only at the caudal level $(\boldsymbol{R})$ indicative of a thinner commissural tract in Vegf ${ }^{\Delta \text { Tiez-Cre }}$ embryos. $n=5$.

the cortical plate (Sturrock, 1981) or very sparsely distributed throughout the cortex (Cunningham et al., 2013). Together, our study signifies an important role for endothelial VEGF in promoting periventricular angiogenesis.

In addition, endothelial VEGF is capable of acting as a key "stop" signal for neuronal proliferation and migration in the embryonic telencephalon. In the absence of endothelial VEGF, overproduction of neuronal progenitors combined with excess neuronal migration in some cases and/or disrupted neuronal mi- gration caused severe malformations in the embryonic telencephalon by late embryonic stages. These findings shed new light on the role of endothelial VEGF in regulating cortical lamination. Moreover, the phenotypic differences resulting from specifically deleting VEGF from neuronal progenitors versus endothelial cells becomes apparent. Although conditional deletion of Vegf from neuronal progenitors resulted in decreased neuronal proliferation, increased apoptosis, and extensive degeneration of the cerebral cortex (Haigh et al., 2003; Raab et al., 2004), in sharp 
contrast, conditional deletion of VEGF from endothelial cells resulted in an abnormal increase of neuronal proliferation and disturbed neuronal migration during cortical development. The overmigration of neurons past the breached pial membrane, radial glial detachment, and formation of MZ heterotopias in Vegf ${ }^{\Delta \text { Tiez-Cre }}$ embryos resembles cobblestone lissencephaly (Olson and Walsh, 2002), one common form of cortical dyslamination seen in human congenital muscular dystrophy syndromes. In addition to the key radial migration event essential for cerebral cortex development (Rakic, 1988; Hatten, 2002), the tangential migration event of GABA neurons (Marin and Rubenstein, 2001) was also affected in Vegf ${ }^{\Delta \text { Tie2-Cre }}$ embryos. Further work is needed to clarify whether this is a result of a loss of GABA neuron populations in the absence of endothelial VEGF or the lack of necessary guidance cues along the tangential route or both. Axonal tracts that follow stereotyped pathways (Lopez-Bendito and Molnar, 2003) were markedly impaired in Vegf $f^{\Delta T i e 2-C r e}$ embryos, bringing forth additional questions as to the precise role of endothelial VEGF in axon growth and guidance. Interestingly, neuronal VEGF cannot compensate for all of these defects caused in the absence of endothelial VEGF.

Lack of endothelial VEGF may contribute to diseases, such as cobblestone lissencephaly, mental retardation, epilepsy, and developmental dyslexia. Our data illustrate the intimate and symbiotic relationship between endothelial cells and neurons of the developing brain that respond meticulously and independently to VEGF signals depending on the cell type that secretes it.

\section{References}

Breier G, Clauss M, Risau W (1995) Coordinate expression of vascular endothelial growth factor receptor-1 (flt-1) and its ligand suggests a paracrine regulation of murine vascular development. Dev Dyn 204:228 -239. CrossRef Medline

Carmeliet P, Ferreira V, Breier G, Pollefeyt S, Kieckens L, Gertsenstein M, Fahrig M, Vandenhoeck A, Harpal K, Eberhardt C, Declercq C, Pawling J, Moons L, Collen D, Risau W, Nagy A (1996) Abnormal blood vessel development and lethality in embryos lacking a single VEGF allele. Nature 380:435-439. CrossRef Medline

Cunningham CL, Martinez-Cerdeno V, Noctor SC (2013) Microglia regulate the number of neural precursor cells in the developing cerebral cortex. J Neurosci 33:4216-4233. CrossRef Medline

Erblich B, Zhu L, Etgen AM, Dobrenis K, Pollard JW (2011) Absence of colony stimulation factor-1 receptor results in loss of microglia, disrupted brain development and olfactory deficits. PLoS One 6:e26317. CrossRef Medline

Fantin A, Vieira JM, Plein A, Denti L, Fruttiger M, Pollard JW, Ruhrberg C (2013) NRP1 acts cell autonomously in endothelium to promote tip cell function during sprouting angiogenesis. Blood 121:2352-2362. CrossRef Medline

Ferrara N, Carver-Moore K, Chen H, Dowd M, Lu L, O'Shea KS, PowellBraxton L, Hillan KJ, Moore MW (1996) Heterozygous embryonic lethality induced by targeted inactivation of the VEGF gene. Nature 380: 439-442. CrossRef Medline

Goossens S, Janzen V, Bartunkova S, Yokomizo T, Drogat B, Crisan M, Haigh K, Seuntjens E, Umans L, Riedt T, Bogaert P, Haenebalcke L, Berx G, Dzierzak E, Huylebroeck D, Haigh JJ (2011) The EMT regulator Zeb2/
Sip1 is essential for murine embryonic hematopoietic stem/progenitor cell differentiation and mobilization. Blood 117:5620-5630. CrossRef Medline

Haigh JJ, Morelli PI, Gerhardt H, Haigh K, Tsien J, Damert A, Miquerol L, Muhlner U, Klein R, Ferrara N, Wagner EF, Betsholtz C, Nagy A (2003) Cortical and retinal defects caused by dosage-dependent reductions in VEGF-A paracrine signaling. Dev Biol 262:225-241. CrossRef Medline

Hatten ME (2002) New directions in neuronal migration. Science 297: 1660-1663. CrossRef Medline

Hicklin DJ, Ellis LM (2005) Role of the vascular endothelial growth factor pathway in tumor growth and angiogenesis. J Clin Oncol 23:1011-1027. CrossRef Medline

Koni PA, Joshi SK, Temann UA, Olson D, Burkly L, Flavell RA (2001) Conditional vascular cell adhesion molecule 1 deletion in mice: impaired lymphocyte migration to bone marrow. J Exp Med 193:741-754. CrossRef Medline

Lee S, Chen TT, Barber CL, Jordan MC, Murdock J, Desai S, Ferrara N, Nagy A, Roos KP, Iruela-Arispe ML (2007) Autocrine VEGF signaling is required for vascular homeostasis. Cell 130:691-703. CrossRef Medline

Lopez-Bendito G, Molnár Z (2003) Thalamocortical development: how are we going to get there? Nat Rev Neurosci 4:276-289. CrossRef Medline

Marín O, Rubenstein JL (2001) A long, remarkable journey: tangential migration in the telencephalon. Nat Rev Neurosci 2:780-790. CrossRef Medline

Mustonen T, Alitalo K (1995) Endothelial receptor tyrosine kinases involved in angiogenesis. J Cell Biol 129:895-898. CrossRef Medline

Ogunshola OO, Stewart WB, Mihalcik V, Solli T, Madri JA, Ment LR (2000) Neuronal VEGF expression correlates with angiogenesis in postnatal developing rat brain. Brain Res Dev Brain Res 119:139-153. CrossRef Medline

Ogunshola OO, Antic A, Donoghue MJ, Fan SY, Kim H, Stewart WB, Madri JA, Ment LR (2002) Paracrine and autocrine functions of neuronal vascular endothelial growth factor (VEGF) in the central nervous system. J Biol Chem 277:11410-11415. CrossRef Medline

Olson EC, Walsh CA (2002) Smooth, rough and upside-down neocortical development. Curr Opin Genet Dev 12:320-327. CrossRef Medline

Raab S, Beck H, Gaumann A, Yüce A, Gerber HP, Plate K, Hammes HP, Ferrara N, Breier G (2004) Impaired brain angiogenesis and neuronal apoptosis induced by conditional homozygous inactivation of vascular endothelial growth factor. Thromb Haemost 91:595-605. CrossRef Medline

Rakic P (1988) Defects of neuronal migration and the pathogenesis of cortical malformations. Prog Brain Res 73:15-37. CrossRef Medline

Schlaeger TM, Bartunkova S, Lawitts JA, Teichmann G, Risau W, Deutsch U, Sato TN (1997) Uniform vascular-endothelial-cell-specific gene expression in both embryonic and adult transgenic mice. Proc Natl Acad Sci U S A 94:3058-3063. CrossRef Medline

Sturrock RR (1981) Microglia in the prenatal mouse neostriatum and spinal cord. J Anat 133:499-512. Medline

Vasudevan A, Long JE, Crandall JE, Rubenstein JL, Bhide PG (2008) Compartment-specific transcription factors orchestrate angiogenesis gradients in the embryonic brain. Nat Neurosci 11:429-439. CrossRef Medline

Virgintino D, Errede M, Robertson D, Girolamo F, Masciandaro A, Bertossi M (2003) VEGF expression is developmentally regulated during human brain angiogenesis. Histochem Cell Biol 119:227-232. Medline

Won C, Lin Z, Kumar TP, Li S, Ding L, Elkhal A, Szabó G, Vasudevan A (2013) Autonomous vascular networks synchronize GABA neuron migration in the embryonic forebrain. Nat Commun 4:2149. CrossRef Medline 